Original paper

\title{
Definition of the margin of major coronary artery bifurcations during radiotherapy with electrocardiograph-gated 4D-CT
}

\author{
Qian Li $^{\mathrm{a}, \mathrm{b}}$, Ying Tong ${ }^{\mathrm{a}, \mathrm{b}}$, Yong Yin ${ }^{\mathrm{a}}$, Pinjing Cheng ${ }^{\mathrm{b}}$, Guanzhong Gong ${ }^{\mathrm{a}, *}$ \\ a Radiation Physics Department of Shandong Cancer Hospital Affiliated to Shandong University, Jinan 250117, China \\ ${ }^{\mathrm{b}}$ School of Nuclear Science and Technology, University of South China, Hengyang 421001, China
}

\section{A R T I C L E I N F O}

\section{Keywords:}

Electrocardiography-gated

Coronary artery bifurcations

Margin

\begin{abstract}
A B S T R A C T
Purpose: The aim was to measure the cardiac motion-induced displacements of major coronary artery bifurcations utilizing electrocardiography (ECG)-gated four-dimensional computed tomography (4D-CT) and to determine the margin of coronary artery bifurcations.

Methods: Thirty-seven female patients who underwent retrospective ECG-gated 4D-CT in inspiratory breath hold (IBH) were enrolled. The left main coronary artery bifurcation (LM), the obtuse marginal branch bifurcation $(O M)$, the first diagonal branch bifurcation $\left(D_{1}\right)$, the second diagonal branch bifurcation $\left(D_{2}\right)$, the caudal portion of the left anterior descending branch (APX), the first right ventricular artery bifurcation (V) and the acute marginal branch bifurcation (AM) were contoured. The center of the contour of the coronary arterial bifurcations at end systole was defined as the standard, and the margin were then calculated.

Results: The margin in the left-right (LR), cranio-caudal (CC), and anterior-posterior (AP) coordinates were as follows: LM 3, 3, and $3 \mathrm{~mm}$; $\mathbf{D}_{\mathbf{1}}$ 6, 3, and $3 \mathrm{~mm}$; $\mathbf{D}_{2}$ 3, 3, and $3 \mathrm{~mm}$; APX 4, 4, and $4 \mathrm{~mm}$; OM 4, 6, and $5 \mathrm{~mm}$; V 6 , 8 , and $7 \mathrm{~mm}$; and AM 6, 8, and $7 \mathrm{~mm}$, respectively.

Conclusion: Coronary artery bifurcations should be considered a separate organ at risk (OAR), and different margin should be provided due to the differences resulting from motion displacement. The maximum margin in the LR, CC, and AP coordinates of left coronary artery bifurcations were 6,6 , and $5 \mathrm{~mm}$, and those of the right coronary artery bifurcations were 6,8 , and $7 \mathrm{~mm}$, respectively.
\end{abstract}

\section{Introduction}

Radiotherapy improves the life expectancy of patients with thoracic malignant tumors; simultaneously, radiation-induced heart disease (RIHD) has been documented with the development of treatment-associated cardiac toxic effects. These effects could cause widespread concern about the problem of RIHD [1-3]. RIHD includes radiationinduced pericarditis, myocarditis, coronary artery disease (CAD), valvular heart disease, and conduction system disease [1-3]. Radiationinduced CAD generally has a long latent period of clinical toxicity and may significantly reduce the quality of patients' survival and even lead to death [4]. Mediastinal and breast radiotherapy involves inadvertent radiation to the heart and coronary artery, increasing the risk of ischemic heart disease [5,6]. Hahn et al. [7] suggested that a dose-volume index of the coronary artery $\left(\mathrm{V}_{5}, \mathrm{~V}_{20}\right)$ was more accurate than the heart mean dose in predicting the risk of ischemic heart disease. Epidemiological studies have revealed that the distribution of coronary atherosclerosis is inhomogeneous in different branches and in different segments of the same branch $[8,9]$. Atherosclerosis plaques were more common near the side branches of coronary arteries, and Givehchi [10] et al. measured the accurate bifurcation angle to determine the correlation with plaque buildup. The development of atherosclerosis is closely related to endothelial cells and blood flow dynamics [11]. The permeability of endothelial cells to macromolecules in regions of arterial branching or curvature is increased, resulting in low-density lipoprotein accumulation and thus the formation of atherosclerosis plaques $[12,13]$. Atherosclerosis is accelerated by radiation in medium to large coronary arteries, and several studies have reported coronary arterial events after radiotherapy. The findings of Fuzellier et al. [14] indicated that the risk of radiation-induced CAD should be considered when the mediastinal radiation dose is greater than or equal to $30 \mathrm{~Gy}$. Coronary artery bifurcations and proximal segments that are located in the irradiation field become preferential sites for lesion formation, whereas distal segments are usually unaffected [15]. The coronary artery is a late-responding organ; as a result of the complexity in delineation and inadequate knowledge of motion, it is not considered a routine organ at risk (OAR) during radiotherapy planning [16]. The marked movements of the coronary artery caused by cardiac motion

\footnotetext{
* Corresponding author.

E-mail address: gongguanzhong@yeah.net (G. Gong).
} 
include stretching, bending and torsion [9]. Organ motion leads to inaccurate contouring results that cause deviations between the planning dose and delivered dose; moreover, the magnitude of coronary motion caused by heartbeat could result in dosimetry variation [17]. Inaccurately contouring an OAR can have severe consequences; hence, it is important for a planner to precisely contour the OAR. To compensate for the difference in doses caused by organ movement, an appropriate margin should be provided for the OAR, and a similar margin should be provided for the coronary artery. To date, there has been no consensus on the study of the coronary artery margin. Different scholars have studied the influence of the setup and penumbra on the margin, while few publications describe the influence of organ motion.

The present study aimed to quantify displacements of coronary artery main bifurcations in the left-right (LR), cranio-caudal (CC), and anterior-posterior (AP) coordinates and concluded that the external distance in the three directions would suggest an appropriate margin for radiotherapy planning.

\section{Methods and materials}

\subsection{Patients and image acquisition}

The study cohort consisted of 37 female patients who underwent retrospective enhanced electrocardiography (ECG)-gated four-dimensional computed tomography (4D-CT). ECG-gated 4D-CT scans were performed during an inspiration breath hold to eliminate the influence of respiration on cardiac motion. Images were obtained for two $\mathrm{R}$ waves and rebuilt at every $5 \%$ interval in the cardiac cycle, and the images were segregated into $0 \%-95 \%$ 20-phase sets with 0.75 -mm slice thicknesses and a 0.5-mm interval (SOMATOM Definition Flash, SIEMENS, Germany).

\subsection{Delineation of coronary artery major bifurcations}

The left main coronary artery bifurcation (LM), the obtuse marginal bifurcation $(\mathrm{OM})$, the first diagonal branch bifurcation $\left(\mathrm{D}_{1}\right)$, the second diagonal branch bifurcation $\left(D_{2}\right)$ and the caudal portion of the left anterior descending branch (APX) in the left coronary artery, the first right ventricular artery bifurcation (V) and the acute marginal bifurcation (AM) in the right coronary artery were selected. Fig. 1 illustrates the selection of coronary bifurcations. The proximal left circumflex segment was defined as the initial segment of the artery before the obtuse marginal branch; the distal left circumflex segment was defined from the obtuse marginal branch to the tip of the circumflex. The proximal, middle, and distal segments of the left anterior
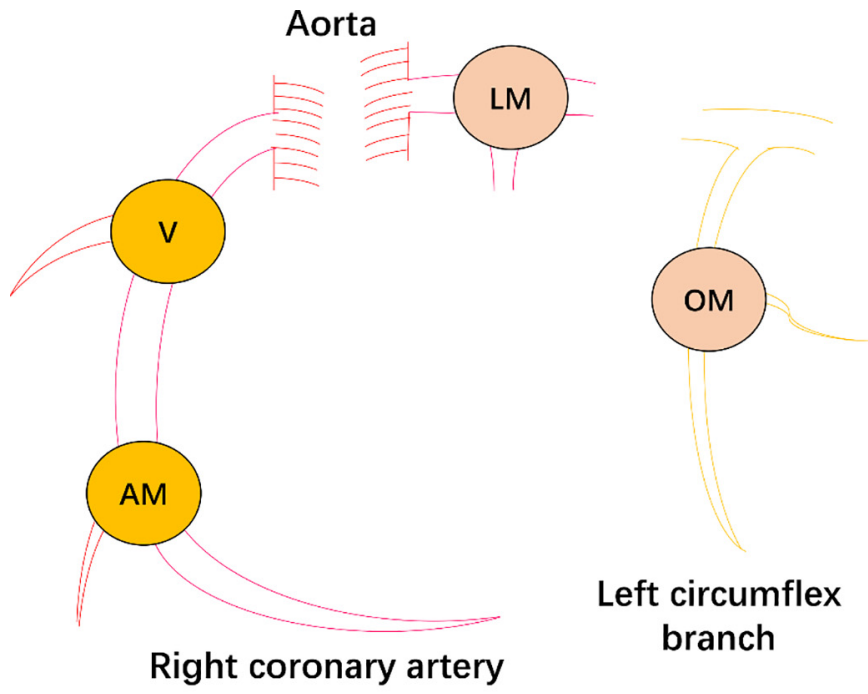

descending (LAD) artery were divided by $\mathrm{D}_{1}$ and $\mathrm{D}_{2}$. The proximal, middle, and distal segments of the right coronary artery were divided by V and AM. All bifurcations were contoured $2 \mathrm{~mm}$ in CC coordinate on the cross-sectional image of the 20 phases using MIM Maestro 6.7.6 software (MIM Software Inc., USA). The software provided the center of the contour of the coronary arterial bifurcations for all three axes.

\subsection{Measurement of bifurcation displacements}

The maximum displacements of every bifurcation in every patient were calculated in three coordinates and were defined as the center of contour of the bifurcations between the maximum and the minimum values. The average maximum displacements were calculated for every bifurcation.

\subsection{Margin calculation}

During a complete cardiac cycle, $25-45 \%$ of the cycle is considered the end-systolic phase. Using the center of the contour of the coronary arterial bifurcation at the end of cardiac systole as the standard, we calculated the displacements of all structures referring to the standard. The means and standard deviations of the major bifurcations displacements of each patient were calculated. The standard deviation of the mean displacements was a systematic error called $\Sigma$, and the rootmean-square of the standard deviation was a random error called $\sigma$. The margin was calculated by the formula $1.3 \Sigma+0.5 \sigma$ [18].

\subsection{Statistical analysis}

Analysis was conducted using SPSS19.0 software; the data are in the form of $\bar{x}+s$.

\section{Results}

The displacements of left coronary arterial bifurcations for the LR, CC, and AP coordinates were as follows: LM 7.5, 6.2, and $6.2 \mathrm{~mm}$; OM $11.8,11.8$, and $10.8 \mathrm{~mm} ; \mathrm{D}_{1} 5.7,6.7$, and $6.5 \mathrm{~mm} ; \mathrm{D}_{2} 5.3,7.1$, and $6.3 \mathrm{~mm}$; and APX 6.4, 7.7, and $4.6 \mathrm{~mm}$, respectively. The displacement of the left circumflex bifurcation OM varied slightly on all three axes. The left circumflex OM had more displacement than the LAD bifurcations $\mathrm{D}_{1}, \mathrm{D}_{2}$ and APX in all three axes. The movement of LAD was predominantly in the $\mathrm{CC}$ coordinate. The proximal and middle segments of LAD bifurcations $\mathrm{D}_{1}$ and $\mathrm{D}_{2}$ had greater AP and less LR displacement than the distal segment bifurcation APX in the LR and CC coordinates.

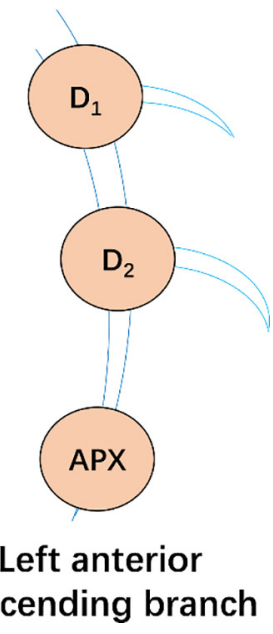

Fig. 1. Coronary artery main bifurcation $(\mathrm{LM}=$ left main coronary artery bifurcation; $\mathrm{OM}=$ obtuse marginal bifurcation; $\mathrm{D}_{1}=$ first diagonal branch bifurcation; $\mathrm{D}_{1}=$ second diagonal branch bifurcation; APX = tip of the left anterior descending branch; $\mathrm{V}=$ first right ventricular artery bifurcation; $\mathrm{AM}=$ acute marginal bifurcation). 


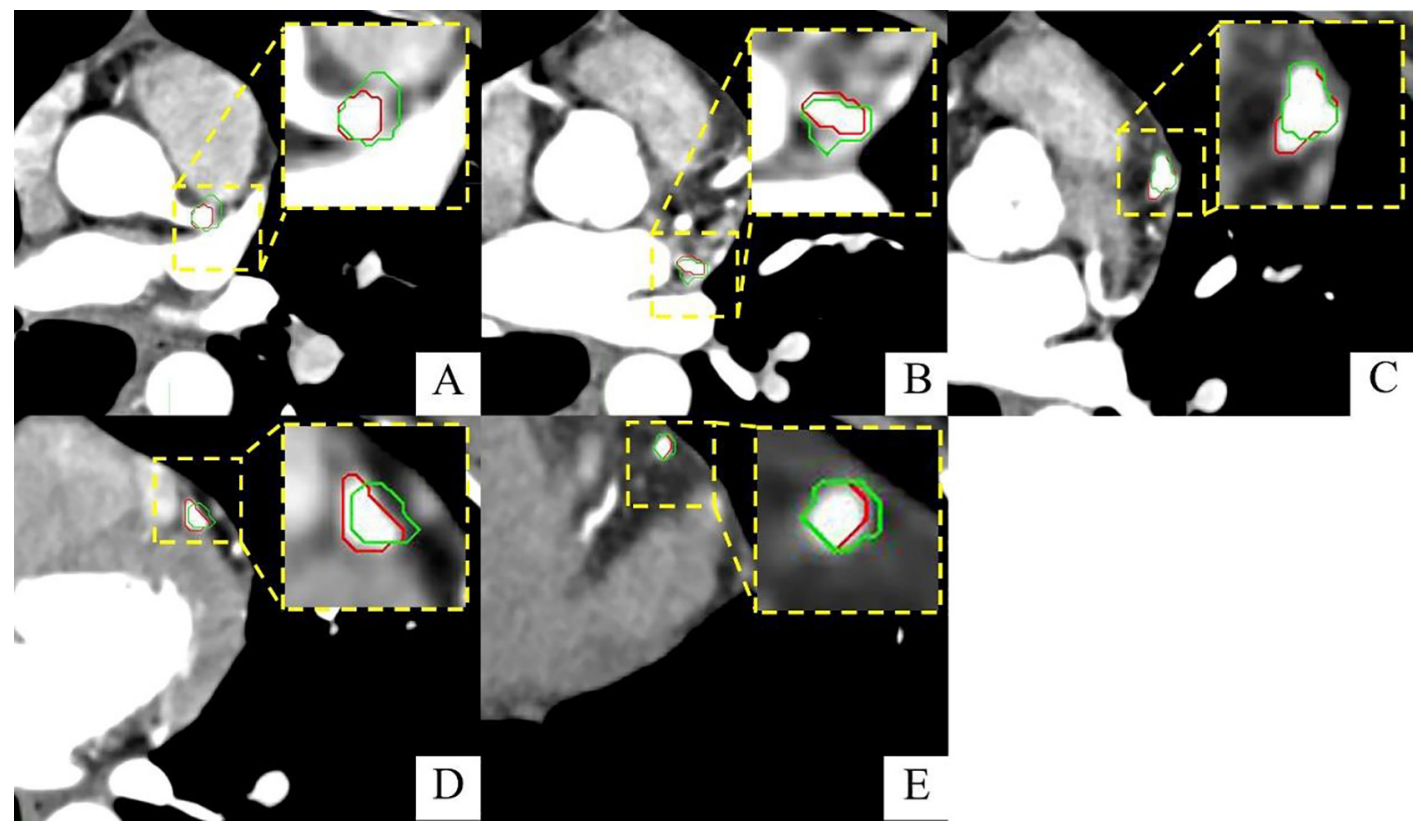

Fig. 2. The $55 \%$ and $70 \%$ phases delineation of left coronary artery bifurcations (A-E were $55 \%$ phase image of LM, OM, $D_{1}, D_{2}$ and $A P X$, respectively. The delineation of LM, OM, $D_{1}, D_{2}$ and APX in $55 \%$ phase were revealed in red, and the delineation of them in $70 \%$ phase were revealed in green).

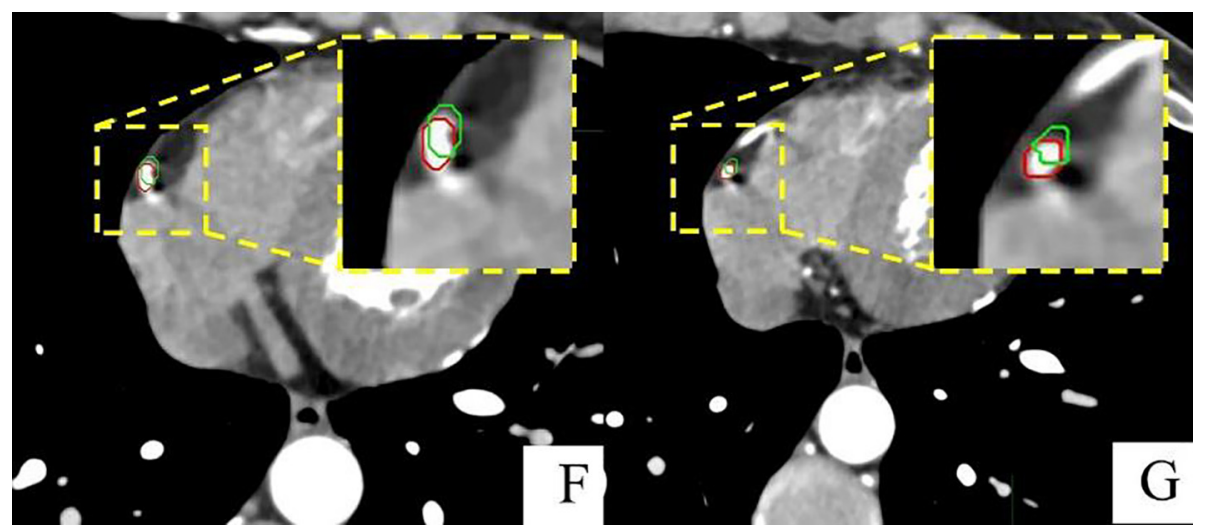

Fig. 3. The $30 \%$ and $35 \%$ phases delineation of right coronary artery bifurcations (F-G were $30 \%$ phase image of $\mathrm{V}$ and AM, respectively. The delineation of $\mathrm{V}$ and $\mathrm{AM}$ in $30 \%$ phase were revealed in red, and the delineation of them in $35 \%$ phase were revealed in green.)

Table 1

Displacements of main coronary artery bifurcations (mm).

\begin{tabular}{llll}
\hline Bifurcations & LR & CC & AP \\
\hline LM & $7.5 \pm 2.6(3.1-13.3)$ & $6.2 \pm 2.0(3.0-11.0)$ & $6.2 \pm 2.3(2.6-12.4)$ \\
OM & $11.8 \pm 4.0(5.1-20.8)$ & $11.8 \pm 5.5(5.0-28.5)$ & $10.8 \pm 4.1(3.5-22.0)$ \\
D $_{1}$ & $5.7 \pm 1.9(3.6-12.1)$ & $6.7 \pm 2.1(3.0-12.4)$ & $6.5 \pm 4.4(2.1-23.8)$ \\
D $_{2}$ & $5.3 \pm 1.9(1.7-9.3)$ & $7.1 \pm 2.5(2.8-18.0)$ & $6.3 \pm 3.6(2.1-22.0)$ \\
APX & $6.4 \pm 3.4(2.4-17.0)$ & $7.7 \pm 3.0(3.4-15.5)$ & $4.6 \pm 2.4(1.2-13.2)$ \\
V & $15.4 \pm 4.0(7.0-22.8)$ & $17.4 \pm 6.1(7.3-31.1)$ & $18.4 \pm 4.9(8.9-30.5)$ \\
AM & $15.4 \pm 3.9(8.1-22.5)$ & $18.7 \pm 6.2(7.0-32.0)$ & $21.8 \pm 4.8(13.9-30.5)$ \\
\hline
\end{tabular}

The displacements of right coronary arterial bifurcations for the LR, CC, and AP coordinates were V 15.4, 17.4, and $18.4 \mathrm{~mm}$, respectively, and AM 15.4, 18.7, and $21.8 \mathrm{~mm}$, respectively. The right coronary arterial bifurcations had obvious displacements in the three coordinates. The displacements were greater for the distal segment of the AM artery bifurcation than the proximal segment of the V artery bifurcation; the displacements of $\mathrm{V}$ and $\mathrm{AM}$ in the LR, CC, and AP coordinates were AP > CC > LR. Fig. 2 showed the $55 \%$ and $70 \%$ phases delineation of left coronary artery bifurcations. Fig. 3 showed the $30 \%$ and $35 \%$ phases delineation of right coronary artery bifurcations. We could observe the motion of coronary bifurcations during the cycle of heartbeat. The displacements of the coronary artery main ostia are shown in Table 1.

The values of $\Sigma$ and $\sigma$ are shown in Table 2. The margin (rounded to the nearest $\mathrm{mm}$ ) of the left coronary arterial bifurcations in the LR, CC, and AP coordinates were LM 3, 3, and $3 \mathrm{~mm}$; OM 4, 6, and $5 \mathrm{~mm} \mathrm{D}_{1} 6$, 3 , and $3 \mathrm{~mm} ; \mathrm{D}_{2} 3,3$, and $3 \mathrm{~mm}$; and $\mathrm{APX} 4,4$, and $4 \mathrm{~mm}$. The left coronary arterial bifurcation margin was homogeneous for the LR, CC, and AP coordinates, while the margin of $\mathrm{D}_{1}$ for the LR coordinates was obviously greater than that for the $\mathrm{CC}$ and AP coordinates. 
Table 2

Value of systematic error $\Sigma$ and radom error $\sigma$.

\begin{tabular}{|c|c|c|c|c|c|c|}
\hline & \multicolumn{3}{|l|}{$\Sigma$} & \multicolumn{3}{|l|}{$\sigma$} \\
\hline & LR & CC & $\mathrm{AP}$ & LR & $\mathrm{CC}$ & $\mathrm{AP}$ \\
\hline LM & 0.1227 & 0.1050 & 0.1046 & 0.2048 & 0.2083 & 0.1991 \\
\hline $\mathrm{OM}$ & 0.1438 & 0.3110 & 0.2510 & 0.3357 & 0.3630 & 0.3163 \\
\hline $\mathrm{D}_{1}$ & 0.3386 & 0.1144 & 0.0832 & 0.1786 & 0.2301 & 0.1919 \\
\hline $\mathrm{D}_{2}$ & 0.1155 & 0.1055 & 0.0983 & 0.1649 & 0.2366 & 0.1908 \\
\hline APX & 0.2207 & 0.1612 & 0.1948 & 0.2115 & 0.2147 & 0.1289 \\
\hline V & 0.2220 & 0.3828 & 0.2485 & 0.4783 & 0.5303 & 0.5919 \\
\hline $\mathrm{AM}$ & 0.1947 & 0.3404 & 0.2635 & 0.4960 & 0.5546 & 0.6829 \\
\hline
\end{tabular}

Table 3

Margin of main coronary artery bifurcations (mm).

\begin{tabular}{llll}
\hline Bifurcations & LR & CC & AP \\
\hline LM & 3 & 3 & 3 \\
OM & 4 & 6 & 5 \\
$\mathrm{D}_{1}$ & 6 & 3 & 3 \\
$\mathrm{D}_{2}$ & 3 & 3 & 3 \\
$\mathrm{APX}$ & 4 & 4 & 4 \\
$\mathrm{~V}$ & 6 & 8 & 7 \\
$\mathrm{AM}$ & 6 & 8 & 7 \\
\hline
\end{tabular}

The margin (rounded to the nearest $\mathrm{mm}$ ) of the right coronary arterial bifurcations for the LR, CC, and AP coordinates were V 6, 8, and $7 \mathrm{~mm}$, respectively and $\mathrm{AM} 6,8$, and $7 \mathrm{~mm}$, respectively. The right coronary arterial bifurcation margin was inhomogeneous for the LR, CC, and AP coordinates, i.e., LR < AP < CC. The margin of the coronary artery main bifurcations are shown in Table 3 .

The standard margin of the coronary artery major bifurcations indicated that the coverage rate of margin was $93 \% \pm 3.2 \%$ (90\%-100\%).

\section{Discussion}

The coronary artery is adjacent to irradiation fields, and its cardiac motion-induced movements are complex; thus, the coronary artery receives a high dose during mediastinum and breast radiotherapy, and severe RIHD can occur. Handler et al. [19] proved that radiotherapy can result in coronary stenosis. Several studies have shown a marked dependency between the risk of coronary artery injury and dose. Darby et al. [5] found that the rate of major coronary events increased linearly with the mean dose to the heart by $7.4 \%$ per gray for breast cancer radiotherapy. Moignier et al. [20] found the rate of major coronary events increased with the mean dose to the coronary artery by $4.9 \%$ per gray in Hodgkin's lymphoma patients who received mediastinal radiation. Our study calculated the displacements of the coronary artery caused by heartbeat and then defined the coronary artery margin, which provided support for the prediction and protection of the radiation-induced injury of the coronary artery in radiation therapy of thoracic tumor, which held clinical significance for future studies.

In the current study, the displacements of the left coronary artery bifurcations were predominantly in the CC coordinate; the right coronary artery bifurcations had obvious AP movement during a complete cardiac cycle. The right coronary artery bifurcations had various displacements for the three coordinates. These findings reminded us that the motion of the coronary arteries caused by cardiac activity is more complex than three-dimensional motion. Johnson et al. [21] observed coronary motion using ECG-gated biplane angiography films. The movement of the left circumflex and LAD were predominantly in the CC coordinate and accounted for $48 \%$ and $59 \%$, respectively, of the movement. The movement of the right coronary artery was predominantly in the LR coordinate, accounting for $48 \%$ of the movement. We observed notable displacements of the LAD bifurcations $\mathrm{D}_{1}, \mathrm{D}_{2}$, and
APX in the CC coordinate, accounting for $35.4 \%, 38.0 \%$ and $41.2 \%$, respectively. The variation in left circumflex bifurcation OM displacements for three coordinates was not obvious. The displacements of the right coronary artery bifurcations were obvious for the AP coordinate, accounting for $35.9 \%$ and $39.0 \%$, respectively. These findings disagreed with our study and may be related to differences in the selection of mark points.

The right coronary artery bifurcations had larger movement than the left coronary artery bifurcations, and the range of left circumflex bifurcation OM motion was at a maximum because the left circumflex and right coronary artery both run though the atrioventricular sulcus in the basal plane of the heart, and studies have shown that cardiac motion increases from the apex to the base [22,23]. Similarly, Lu et al. [24] concluded that the left circumflex and right coronary artery had the same movement characteristics, which was consistent with our findings. Kataria et al. [16] calculated the average displacement at end systole and end diastole for the LR, CC, and AP coordinates; specifically, the LAD displacements were $3.0 \pm 1.6,2.8 \pm 1.5$, and $3.6 \pm 2.0 \mathrm{~mm}$, respectively, the left circumflex displacements were $4.9 \pm 1.6,2.9 \pm 1.3$, and $5.1 \pm 1.9 \mathrm{~mm}$, respectively, and the right coronary artery displacements were $6.6 \pm 2.2,3.6 \pm 2.1$, and $5.9 \pm 2.2 \mathrm{~mm}$, respectively. The displacements of bifurcations that we observed were larger than these values, likely due to an averaging effect. The overall movement of the coronary arteries is not as significant as that of the bifurcations.

There are both systematic and random errors in the processes of radiotherapy preparation and delivery, such as uncertainty in CTguided laser aiming, setup error, and organ motion[18][25]. In the present study, the formula for margin calculation mainly referred to the methods proposed by McKenzie[18] et al. Systematic errors are persistent, while random errors have more uncertainty. Therefore, it is necessary to consider both types of error when calculating the margin. The movement of the heart is repeated cyclically in three dimensions, and the movement displacement of each axis is consistent with the Gaussian distribution. The dose distribution was shifted closer to or further away from an organ at risk due to systematic errors, and the magnitude $\Sigma$ determines the feasibility of the coronary margin encompassing the coronary mean position during radiotherapy[18]. McKenzie et al. [18] thought that adding $1.3 \Sigma$ in width to the margin of the OAR could ensure inclusion of $90 \%$ the mean position of the OAR in any single direction. The coronary effects of the random error caused by organ movement and setup errors during radiotherapy may lead to serious radiation-induced damage [26]. Thus, the prudent course of action was to add a $0.5 \sigma$ coronary margin to the systematic margin to control the coronary dose within a safe range [18].

In the present study, we found that the coverage rate of the margin was $93 \% \pm 3.2 \%$, which was superior to the study by McKenzie et al. [18]. The coverage of the major bifurcation margin in the CC direction shows a slight advantage compared with the coverages obtained in the LR and AP directions for two reasons; specifically, the cardiac motioninduced coronary movement in the CC direction is more complicated and the main bifurcations of the coronary artery were delineated only at $2 \mathrm{~mm}$ in the CC direction in this study, resulting in the calculation of a small bifurcation margin in the CC direction.

The LAD is adjacent to the radiation field during mediastinal and breast radiotherapy; thus, the margin of the LAD should be more conservative [27]. Taylor et al. [28] reported that the dose varied among different LAD segments for left breast radiotherapy; the dose at the proximal segment was less than $4 \mathrm{~Gy}(10 \%$ of the tumor dose) because outside of the field, the middle segment and the distal segment were affected by the tangential beam, and the distal segment dose was greater than $30 \mathrm{~Gy}$ ( $75 \%$ of the tumor dose). As a result of the various doses among different segments of the LAD, the margin of bifurcations should be diverse. In the present study, the margin of LAD bifurcations increased from $D_{1}, D_{2}$ to APX in the left coronary artery, and the margin of $\mathrm{D}_{2}$ and APX is homogeneous for the three coordinates. The margin of 
$\mathrm{D}_{1}$ for the LR coordinate was clearly more than that of the CC and AP coordinates. This finding may be related to the individual differences in the diagonal branches that are usually $3-5$ branches, and sometimes the first diagonal branch comes directly from the left main coronary artery.

Few publications describe the contribution of cardiac activity to coronary artery movement; those that do have mostly focused on the respiratory activity that affects the heart and the cardiac substructure movement. The main reason lies in the fact that the coronary artery has irregular lumens, quantifying coronary artery movement afterward is difficult, and the study of the respiration activity that induces coronary motion cannot eliminate the influence of cardiac activity. Thus, some conclusions need further confirmation. Studying the margin of major bifurcations based on a respiratory and electrocardiography-gated technique is more accurate. However, the time of the single phase in the respiratory-gated technique is notably longer than that in the electrocardiography-gated technique. Therefore, dividing the cardiac cycle completely with the respiratory gate is difficult. Furthermore, there are no reports of the combination of these two gating technologies online. In this study, all patients were scanned while performing an inspiration breath hold to minimize the influence of respiration on coronary motion. The scanning time of conventional 3D-CT is longer, and confirming the specific phase of the coronary artery in various slices during the cardiac cycle is difficult. 4D-CT was usually used to estimate the internal organ motion [29]. For ECG-gated 4D-CT, the method of volume scanning combined with cardiac cycle tracking was adopted, and the images were analyzed retrospectively based on the electrocardiography curve. The temporal resolution of a single phase in the ECG-gated sequence is sufficient for this study, resulting in high quality with minimal blurring and increasing the accuracy of all bifurcation data, and this information cannot be obtained by 3D-CT.

Rigorous dose constraint and optimization of the radiotherapy plan design and delivery can reduce the risk of RIHD; these conditions are closely related to the accurate delineation of the organs and the use of an appropriate margin for the organs. In this study, the proposed margin should also be constantly adjusted and optimized in practice to protect coronary artery main bifurcations to avoid high-dose irradiation.

\section{Conclusion}

In summary, there are significant differences among the bifurcation displacements; thus, varying margin should be provided for coronary artery bifurcations. The maximum margin in the LR, CC, and AP coordinates of the left coronary artery bifurcations were 6,6 , and $5 \mathrm{~mm}$, respectively, and those for the right coronary artery bifurcations were 6,8 , and $7 \mathrm{~mm}$, respectively.

\section{References}

[1] Zhuang XF, Yang YM, Sun XL, Liao ZK, Huang J. Late onset radiation-induced constrictive pericarditis and cardiomyopathy after radiotherapy: a case report. Medicine 2017;96(5):e5932.

[2] Umezawa R, Takanami K, Kadoya N, Nakajima Y, Saito M, Ota H, et al. Assessment of myocardial metabolic disorder associated with mediastinal radiotherapy for esophageal cancer -a pilot study. Radiat Oncol 2015;10(1):96.

[3] Macgowan GA. The challenge of radiation-induced restrictive cardiomyopathy and outcomes after heart transplantation. J Card Fail 2016;22(6):479-80.

[4] Miltényi Z, Keresztes K, Garai I, Édes I, Galajda Z, Tóth L, et al. Radiation-induced coronary artery disease in Hodgkin's disease. Cardiovasc Radiat Med
2004;5(1):38-43.

[5] Darby SC, Ewertz M, McGale P, Bennet AM, Blom-Goldman U, Brønnum D, et al. Risk of ischemic heart disease in women after radiotherapy for breast cancer. N Engl J Med 2013;368(11):987-98.

[6] Nguyen NP, Krafft SP, Vinh-Hung V, Vos P, Almeida F, Jang S, et al. Feasibility of tomotherapy to reduce normal lung and cardiac toxicity for distal esophageal cancer compared to three-dimensional radiotherapy. Radiother Oncol 2011;101(3):438-42.

[7] Hahn E, Jiang H, Ng A, Bashir S, Ahmed S, Tsang R, et al. Late cardiac toxicity after mediastinal radiation therapy for hodgkin lymphoma: contributions of coronary artery and whole heart dose-volume variables to risk prediction. Int J Radiat Oncol Biol Phys 2017;98(5):1116-23.

[8] Hui Z, Ding Z, Piana RN, Gehrig TR, Friedman MH. Cataloguing the geometry of the human coronary arteries: a potential tool for predicting risk of coronary artery disease. Int J Cardiol 2009;135(1):43-52.

[9] Ding Z, Friedman MH. Dynamics of human coronary arterial motion and its potential role in coronary atherogenesis. J Biomech Eng 2000;122(5):488-92.

[10] Givehchi S, Safari MJ, Tan SK, Md Shah MNB, Sani FBM, Azman RR, et al. Measurement of coronary bifurcation angle with coronary CT angiography: a phantom study. Phys Med 2018;45:198-204.

[11] Sawaya FJ, Lefèvre T, Chevalier B, Garot P, Hovasse T, Morice MC, et al. Contemporary approach to coronary bifurcation lesion treatment. JACC Cardiovasc Interv 2016;9(18):1861-78.

[12] Lusis AJ. Atherosclerosis. Nature 2000;407(6801):233-41.

[13] Mandal SR, Bharati A, Haghighi RR, Arava S, Ray R, Jagia P, et al. Non-invasive characterization of coronary artery atherosclerotic plaque using dual energy CT: explanation in ex-vivo samples. Phys Med 2018;45:52-8.

[14] Fuzellier JF, Mauran P, Metz D. Radiation-induced bilateral coronary ostial stenosis in a 17-year-old patient. J Card Surg 2006;21(6):600-2.

[15] Korosoglou G, Kristen AV, Andrassy M, Katus HA, Hardt SE. Severe left main coronary stenosis in a young female patient, 6 years after mediastinal radiation therapy for non-Hodgkin lymphoma: assessment by coronary angiography and intravascular ultrasound. Clin Res Cardiol 2012;101(4):317-20.

[16] Kataria T, Bisht SS, Gupta D, Abhishek A, Basu T, Narang K, et al. Quantification of coronary artery motion and internal risk volume from ECG gated radiotherapy planning scans. Radiother Oncol 2016;121(1):59-63.

[17] Chen B, Hu Y, Liu J, Cao AN, Ye LX, Zeng ZC. Respiratory motion of adrenal gland metastases: Analyses using four-dimensional computed tomography images. Phys Med 2017;38:54-8.

[18] McKenzie A, van Herk M, Mijnheer B. Margins for geometric uncertainty around organs at risk in radiotherapy. Radiother Oncol 2002;62(3):299-307.

[19] Handler CE, Livesey S, Lawton PA. Coronary ostial stenosis after radiotherapy: angioplasty or coronary artery surgery? Br Heart J 1989;61(2):208-11.

[20] Moignier A, Broggio D, Derreumaux S, Beaudré A, Girinsky T, Paul J, et al. Coronary stenosis risk analysis following Hodgkin lymphoma radiotherapy: a study based on patient specific artery segments dose calculation. Radiother Oncol 2015;117(3):467-72.

[21] Johnson KR, Patel SJ, Whigham A, Hakim A, Pettigrew RI, Oshinski JN. Threedimensional, time-resolved motion of the coronary arteries. J Cardiovasc Magn Reson 2004;6(3):663-73.

[22] Shechter G, Resar JR, Mcveigh ER. Displacement and velocity of the coronary arteries: cardiac and respiratory motion. IEEE Trans Med Imaging 2006;25(3):369-75.

[23] Wang Y, Vidan E, Bergman GW. Cardiac motion of coronary arteries: variability in the rest period and implications for coronary MR angiography. Radiology 1999;213(3):751-8.

[24] Lu B, Mao SS, Zhuang N, Bakhsheshi H, Yamamoto H, Takasu J, et al. Coronary artery motion during the cardiac cycle and optimal ECG triggering for coronary artery imaging. Invest Radiol 2001;36(5):250-6.

[25] Herk MV. Error and margins in radiotherapy. Semin Radiat Oncol 2004;14(1):52-64.

[26] Zhang M, Zhang Q, Gan H, Li S, Zhou SM. Setup uncertainties in linear accelerator based stereotactic radiosurgery and a derivation of the corresponding setup margin for treatment planning. Phys Med 2016;32(2):379-85.

[27] Dombe DD, Anitha T, Giri PA, Dombe SD, Ambiye MV. Clinically relevant morphometric analysis of left coronary artery. Int J Biol Med Res 2012;3(6):717-38.

[28] Taylor CW, Povall JM, McGale P, Nisbet A, Dodwell D, Smith JT, et al. Cardiac dose from tangential breast cancer radiotherapy in the year 2006. Int J Radiat Oncol Biol Phys 2008;72(2):501-7.

[29] Harada K, Katoh N, Suzuki R, Ito YM, Shimizu S, Onimaru R, et al. Evaluation of the motion of lung tumors during stereotactic body radiation therapy (SBRT) with fourdimensional computed tomography (4DCT) using real-time tumor-tracking radiotherapy system (RTRT). Phys Med 2016;32(2):305-11. 